\title{
Movilidad Social y Educación. Creencias sobre obtención y administración de rentas asociadas a las variables socioeconómicas de los estudiantes de Contaduría Pública de la Universidad de La Salle
}

\author{
Social Mobility and Education. Beliefs about obtaining and managing incomes \\ associated with the socioeconomic variables of the students of Public Accounting \\ of the University of La Salle
}

Recibido: 06-06-2018 • Aprobado: 06-11-2018 • Página inicial: 89 - Página final: 111

\author{
Diego Alejandro Peralta Borray* \\ Fabiola Loaiza Robles** \\ Camilo José Tamayo Borray***
}

\begin{abstract}
Resumen: la presente investigación profundiza en la relación que existe entre la educación y los niveles de movilidad social. Con base en los postulados de la sociología del conocimiento, propone que la formación universitaria, como parte de la socialización secundaria de los estudiantes, modifica las actitudes relacionadas con la obtención y administración de rentas como factor que legitima la desigualdad, por tal razón, se analiza el componente cognitivo de la actitud. El estudio se realiza con estudiantes de contaduría pública de la Universidad de La Salle, quienes configuran un contexto social dentro del cual se preparan para afrontar problemas relacionados con el control de la riqueza. El estudio encontró que hay claros niveles de asociación entre las categorías de creencias analizadas, los años de formación y los estratos socioeconómicos de los estudiantes, lo que permite establecer tendencias hacia actitudes específicas de los individuos.
\end{abstract}

Palabras clave: movilidad social, educación universitaria, creencias, educación contable.

\begin{abstract}
Abstrat: The present investigation deepens in the relationship that exists between education and the levels of social mobility. Based on the postulates of the sociology of knowledge, proposes that the university education, as part of the socialization secondary school, modifies attitudes related to obtaining and administering income as a factor that legitimizes inequality, for For this reason, the cognitive component of the attitude. The study is carried out with students from public accounting of the University of La Salle, who configure a social context within which prepare to face related problems with the control of wealth. The study found that There are clear levels of association between categories of beliefs analyzed, the years of training and the socioeconomic strata of the students, what that allows to establish tendencies towards attitudes specific to individuals.
\end{abstract}

Keywords: Social mobility, education university, beliefs, accounting education.

* Contador Público y Magíster en Administración de Empresas de la Universidad Nacional de Colombia. Profesor Investigador de la Facultad de Ciencias Administrativas y Contables de la Universidad de La Salle. Integrante del grupo de investigación Sigma Empresarial. daperalta@unisalle.edu.co / alejandroborray@gmail.com Enlace ORCID: https://orcid.org/0000-0003-3410-5970

** Contadora Pública de la Universidad de Manizales y Magíster en Educación y desarrollo humano del Centro Internacional de Educación y Desarrollo Humano - CINDE en convenio con la Universidad de Manizales. Profesora Investigadora de la Facultad de Ciencias Administrativas y Contables de la Universidad de La Salle. Integrante del grupo de investigación Gestión, aministración y organizaciones-GAO. floaiza@unisalle.edu.co / floaizar@gmail.com Enlace ORCID: https://orcid.org/0000-0002-5562-5134

*** Psicólogo y Magister en psicología del consumidor de la Universidad Konrad Lorenz. Consultor y asesor en psicología del consumidor. cajotabo@yahoo.es

Enlace ORCID: https://orcid.org/0000-0003-1385-3777 


\section{Mobilité sociale et éducation. Croyances sur l'obtention et la gestion des revenus associé aux variables socio-économiques des étudiants de comptabilité publique de I’Université de La Salle}

Résume: la présente enquête s'approfondit la relation qui existe entre l'éducation et les niveaux de la mobilité sociale. Basé sur les postulats de la sociologie de la connaissance, propose que le enseignement universitaire, dans le cadre de la socialisation école secondaire, modifie les attitudes liés à l'obtention et à l'administration le revenu en tant que facteur légitimant l'inégalité, Pour cette raison, la composante cognitive de l'attitude. L'étude est réalisée avec des étudiants de comptabilité publique de l'Université de La Salle, qui configurent un contexte social dans lequel se préparer à faire face aux problèmes connexes avec le contrôle de la richesse. L'étude a révélé que Il existe des niveaux clairs d'association entre les catégories des croyances analysées, les années de formation et les couches socio-économiques des étudiants, quelle cela permet d'établir des tendances vers des attitudes spécifique aux individus.

Mots-clés: mobilité sociale, éducation université, croyances, éducation comptable.

\section{Mobilidade Social e Educação. Crenças sobre a obtenção e gestão de rendimentos associados às variáveis socioeconômicas dos estudantes de Contabilidade Pública da Universidade de La Salle}

Resumo: a presente investigação aprofunda-se em a relação que existe entre a educação e os níveis de mobilidade social. Baseado nos postulados da sociologia do conhecimento, propõe que o educação universitária, como parte da socialização escola secundária, modifica atitudes relacionados à obtenção e administração de renda como fator legitimador da desigualdade, por Por essa razão, o componente cognitivo da a atitude. O estudo é realizado com estudantes de contabilidade pública da Universidade de La Salle, que configuram um contexto social dentro do qual prepare-se para enfrentar problemas relacionados com o controle da riqueza. $\mathrm{O}$ estudo descobriu que Existem níveis claros de associação entre categorias das crenças analisadas, os anos de treinamento e os estratos socioeconômicos dos estudantes, o que que permite estabelecer tendências para atitudes específico para os indivíduos.

Palavras-chave: mobilidade social, educação universidade, crenças, educação contábil. 


\section{Introducción}

En mayo de 2014, el Fondo Monetario Internacional publicó la nota de discusión Redistribution, Inequality, and Growth donde se desvirtuaba la postura según la cual altos niveles de desigualdad mejoraban el crecimiento económico. Por el contrario, Ostry, Berg y Tsangarides (2014) concluyeron que una "menor desigualdad neta parece impulsar un crecimiento más rápido y más duradero para un nivel dado de redistribución" (p.56).

Adicionalmente, niveles altos de desigualdad en la distribución de la riqueza conllevan problemas para la formación de capital humano, lo que genera baja productividad y violencia. Por lo tanto, la construcción de una estructura social igualitaria, además de ser uno de los objetivos de la actuación del Estado en su función de redistribución de la riqueza, ${ }^{1}$ requiere la realización de acciones del conjunto de los actores sociales.

Una estructura social "es igualitaria cuando los diferenciales entre los niveles socioeconómicos de la sociedad se desplazan gradualmente hacia niveles de educación e ingreso más homogéneos" (Esteban y Grillo, 2000, p.104). La desigualdad en términos de la distribución asimétrica de la riqueza puede explicarse por la forma desigual en que se distribuyen las rentas (ingreso). Lo anterior, teniendo en cuenta que "la riqueza de las personas es fruto de la acumulación pasada de alguna clase de rentas, principalmente la renta del trabajo, y por ello la desigualdad en renta es el componente fundamental para entender la forma en la que está distribuida la riqueza" (Ríos, 2002, p.222).

Para cumplir con el propósito de distribuir de forma igualitaria la renta, adquiere relevancia la función redistributiva de la educación, en el sentido de permitir al más educado acceso a rentas laborales más altas,

(...) pues preparan para los diferentes roles de la división social del trabajo y asignan el talento de manera eficiente con base en la competencia de los más hábiles. Mayor educación genera mayor productividad potencial de la fuerza de trabajo, y como consecuencia, mayores ingresos potenciales para los trabajadores (Bazdresch, 2001, p.68).

Por otro lado, el estudio realizado por Esteban y Grillo (2000) presenta como condicionantes para mejorar la probabilidad de movilidad social ascendente,

${ }^{1}$ Musgrave (1983), plantea tres funciones para la actuación pública: estabilización, redistribución de la riqueza y provisión de bienes públicos. 


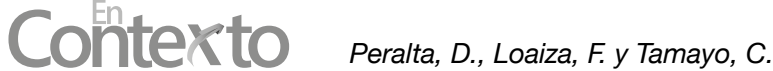

el hecho que los hijos superaran los niveles de educación de los padres. Es importante resaltar que la movilidad social en este estudio se refiere a "el conjunto de cambios de posición (estado) social que se observan básicamente en las variaciones (ascendentes o descendentes) del nivel de educación, de la ocupación, de los ingresos o la riqueza y de los niveles socioeconómicos" (p.104).

Por lo tanto, deben incorporarse dos elementos al análisis económico de la desigualdad: el primero se refiere al factor hereditario, comprobado por Esteban y Grillo (2000), en donde la educación y habilidades de los padres se transmiten a los hijos. Y el segundo en relación con las habilidades adquiridas por parte de los hijos a través de la educación.

Lo anterior coincide con los postulados de Berger y Luckmann (2003), quienes proponen la existencia de dos tipos de socializaciones que configuran la construcción del mundo de los individuos: la primaria, en donde "el niño de clase baja no solo absorbe el mundo social en una perspectiva de clase baja, sino que lo absorbe con la coloración idiosincrásica que le han dado sus padres" (Berger y Luckmann, 2003, p.165). Y la secundaria, donde se da "la adquisición del conocimiento específico de 'roles', estando éstos directa o indirectamente arraigados en la división del trabajo" (Berger y Luckmann, 2003, p.173).

Al nacer, las personas hacen parte de un mundo objetivo o escenario social donde sus conductas son observables, este influye en el espacio vital o mundo psicológico subjetivo del individuo y entre ambos determinan su libre movimiento en el espacio (Lewin, 1978; Berger y Luckmann, 2003). La conducta observable que ocurre en medio de una realidad socialmente construida y que está determinada por aspectos subjetivos y objetivos. (Lewin, 1978; Berger y Luckmann, 2003).

La socialización secundaria es la encargada de internalizar submundos institucionales. La construcción de la realidad subjetiva tiene como marco de referencia una estructura social objetiva aprendida a través de los procesos de socialización, en donde el conocimiento apropiado por el individuo es el resultado del proceso dialéctico individuo-realidad, mediado por la distribución que se da en la sociedad del conocimiento en relación con los distintos subuniversos o submundos institucionales. 
De esta forma, individuos con niveles socioeconómicos diferentes tenderán a construir actitudes ${ }^{2}$ distintas con relación a la producción y administración de rentas, de la misma manera que individuos inmersos en procesos de formación especializada las modificarán para manifestar comportamientos acordes con el sub-universo que se está aprendiendo, "institucionalizando un área de conducta a través del lenguaje y produciendo un tipo específico de individuo" (Berger y Luckmann, 2003).

Toda actividad humana, lo que incluye las profesionales-laborales, está sujeta a la habituación (Berger y Luckmann, 2003). En las relaciones sociales de producción y consumo se identifican dos tipos de hábitos: los relacionados con necesidades, las cuales aportan tensión, ya que son una fuente intrínseca de energía, y los de repetición, que se dan debido a fuerzas restrictivas que determinan un sendero (Lewin, 1978). El conocimiento de la naturaleza permite especificar un procedimiento que al ejecutarse funciona (Berger y Luckmann, 2003) o se puede probar en la práctica, lo que implica observar los cambios esperados en los objetos para comprobar su verdad, y al volverse habitual, tal procedimiento ejecutado provee una especialización diferente a las que vienen por naturaleza en el hombre (Berger y Luckmann, 2003). Esta interacción con el mundo físico y social lleva a un cambio en la estructura cognitiva (Lewin, 1978).

Las universidades, como instituciones donde ocurre la socialización secundaria, tienen la potencialidad de influir en la actitud de sus estudiantes, de tal forma que sea positiva hacia los comportamientos que se considera contribuyen a un mayor desarrollo económico de la sociedad en su conjunto. El campo académico de los estudiantes universitarios, por ejemplo, los de contaduría, configura un contexto social dentro del cual se preparan mediante la adquisición de conocimientos, para afrontar problemas relacionados con la obtención y administración de la riqueza, que ocurrirán en su vida laboral (Suárez, 2015). Por ejemplo, si tienen una actitud negativa hacia la corrupción es posible que el estudiante evite usarla y la considere una forma injusta para obtener rentas que se debe solucionar o mitigar.

En estricto sentido, sería deseable que la educación universitaria contribuyera a fortalecer actitudes que permitan mejorar los niveles de movilidad social, sin embargo, no se tiene claridad si esto está ocurriendo. Tampoco puede afirmarse que las universidades han identificado las actitudes que deben fortalecerse para permitir que estudiantes de niveles sociales bajos tengan las mismas capacidades de capitalizar las oportunidades que los estudiantes de niveles socioeconómicos altos. ${ }^{2}$ La actitud ha sido estudiada por la psicología social (Araya, 2002) (Morales, 1999). El interés en ella radica en que predisponen a
ejecutar determinados comportamientos, que están es intercomunicación e interacción recíproca (Arnau, 2010). 


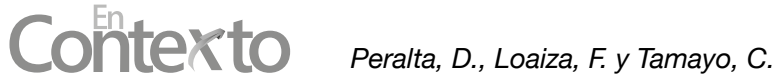

Esto se relaciona con la crítica principal a la mencionada función redistributiva de la educación que "consiste en que, aunque no se lo quiera así, de hecho, las prácticas docentes y los contenidos curriculares son diferentes según la clase social de los alumnos, lo cual reproduce la desigualdad, y más aún, la legitima" (Bazdresch, 2001, p.68).

Esta investigación analizó las actitudes en relación con la obtención y administración de rentas como factor que legitima la desigualdad, con el fin de identificar cuáles se asocian a estudiantes de niveles socioeconómicos altos, y observar si la formación en contaduría pública de la Universidad de La Salle contribuye a fortalecer aquellas que podrían mejorar las posibilidades de movilidad social en estudiantes de niveles socioeconómicos bajos.

La actitud implica la existencia de un objeto ya constituido hacia el cual se reacciona según el tipo de disposición interna que se haya construido hacia él (...) La respuesta que las personas dan ante un objeto determinado está prefigurada ya en la forma en que construyen ese objeto (...) la actitud (...) orienta la respuesta frente a cierto estímulo. (Araya, 2002)

Para este efecto, se entiende que la actitud hace parte del mundo psicológico que el estudiante ha venido formando en interacción con su entorno familiar, educativo y social, ya que ha sido entendida como una disposición interna (Araya, 2002) o estado interno (Morales, 1999), y puede considerarse uno de los factores que facilitan o desalientan el acceso a diferentes zonas y herramientas físicas o sociales para la solución de problemas de la vida cotidiana (Lewin, 1978).

La evaluación que hace alguien de un objeto depende de lo que piensa sobreél (...) el conocimiento que hayamos adquirido acerca de un objeto actitudinal es la base para estimar la evaluación actual del mismo. La experiencia, la observación de la interacción de otros con el objeto o la comunicación, son formas de adquisición de tal conocimiento ${ }^{3}$ (Morales, 1999).

La actitud se define por medio de tres componentes: cognitivo, afectivo y conductual (Morales, 1999; Arnau y Montané, 2010; Ortega, 1986). Sin embargo, hay posiciones contradictorias en cuanto al componente afectivo (Morales, 1999). El componente cognitivo también es llamado creencia (Morales, 1999) u opinión (Suárez, 2015).

${ }^{3}$ El conocimiento se refiere a "la certidumbre de que los fenómenos son reales y que poseen características específicas" (Berger \& Luckmann, 2003). 
El contenido de una creencia puede: a) describir el objeto de la creencia como verdadero o falso, correcto o incorrecto; b) evaluarlo como bueno o malo o, c) propugnar un cierto curso de acción o un cierto estado de existencia como indeseable. Entre creencia y actuación existe una relación, pero no como causa-efecto, sino como tendencia, predisposición o prescripción, en el sentido de orientación o norma para la acción (Suárez, 2015).

Respecto al afecto, se entiende que son estados de ánimo que se mantienen de forma más duradera que una emoción haciendo parte de la experiencia diaria de una persona, tiene una valencia positiva o negativa. El afecto pertenece a estados de ánimo y también a "amplios sistemas cognitivos, motivacionales y de comportamiento" (Clark et al., 1994, citados en Reeve, 2003), además tiene relación con aspectos psico-fisiológicos (Wise, 1996; Ashby et al., 1994, citados en Reeve, 2003).

La aparición de estados afectivos se caracteriza por cambios biológicos que energizan y dirigen acciones corporales con el potencial de provocar conductas observables usadas para adaptarse o enfrentar las situaciones relevantes. Por lo anterior, su medición requiere instrumentos que permitan el registro de variables psicofisiológicas durante largos periodos. Debido a esta restricción, en esta investigación no se evaluó el componente afectivo de la actitud.

Hay evidencia en la vida cotidiana acerca de los antecedentes conductuales de la actitud, en lo que respecta a la formación que las instituciones de diferentes sectores, entre los cuales está el educativo, dan a novatos, aprendices o candidatos (Morales, 1999). Consiste en usar adiestramientos para hacer que la/el novata/o repita frecuentemente las conductas que busca la institución hasta que no haya resistencia, de tal forma que lleguen a formar parte de su repertorio conductual. "Si el adiestramiento culmina adecuadamente, se consigue, en los recién llegados, la creación de esas actitudes que la institución deportiva, religiosa, militar, educativa o del tipo que sea, iba buscando" (Morales, 1999). Se han documentado cambios de actitud en prisioneros de guerra estadounidenses, que luego de su cautiverio decidieron quedarse voluntariamente con los chinos, quienes eran sus enemigos en la Guerra de Corea (Morales, 1999).

El componente conductual exige la observación de conductas en el desarrollo de las actividades concretas de los individuos como técnica de recolección de información, es decir, en el desempeño laboral de los egresados en situaciones naturales o simuladas, las cuales escapan al alcance de esta investigación. 


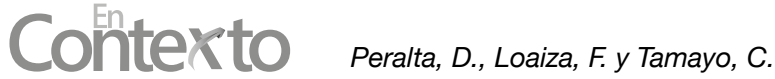

Por este motivo, el componente conductual no se evaluará en este trabajo, que se enfocará únicamente en el componente cognitivo de la actitud, el cual se considera más acorde con el propósito de formación de la educación universitaria.

\section{Metodología}

Esta investigación analizó las actitudes en relación con la obtención y administración de rentas como factor que legitima la desigualdad. Este análisis se realizó a través de la asociación del componente cognitivo de la actitud con variables socioeconómicas de los individuos, para lo cual se usaron cuestionarios que permitieron recolectar la evidencia de la posición evaluativa de la persona frente al objeto actitudinal, e información socioeconómica sobre edad, género, personas a cargo, estado civil, tipo de vivienda, años de educación, área de formación, ocupación, ingreso mensual y estrato socioeconómico. El análisis de la información obtenida se realizó por el método de correspondencias múltiples, en el paquete Factominer del programa estadístico R (R Core Team, 2017).

Las preguntas se enmarcaron en tres categorías de creencias: 1) interacción social; 2) obtención y administración de los ingresos, y 3) actuación ética. Estas categorías se construyeron a través del diálogo con grupos de individuos pertenecientes a la población estudiada, dando origen a las opciones de respuestas cerradas que se usaron en el cuestionario.

La complejidad social impide que se construya metodológicamente un experimento que permita validar deteminada postura en todas sus dimensiones, no obstante, es posible realizar una exploración a través de indagaciones en poblaciones pequeñas de individuos. Para el caso particular de esta investigación se aplicó como técnica el cuestionario, con estudiantes de la carrera de Contaduría Pública de la Universidad de la Salle en Bogotá, la población definida fueron todos los estudiantes de primero, quinto y décimo semestres, con la intención de observar tendencias de asociación de variables relacionadas con la obtención y administración de rentas y su modificación, producto del proceso de formación universitaria de pregrado en un área de las ciencias económicas.

\section{Resultados}

El cuestionario fue respondido por 123 estudiantes, 55 de primer semestre, 37 de quinto semestre y 31 de décimo semestre. En la Tabla 1 se relacionan los descriptivos de cada grupo y en la Tabla 2, los descriptivos de las categorías evaluadas. 
Tabla 1.

Descriptivos de Variables Socioeconómicas

\begin{tabular}{|c|c|c|c|c|c|}
\hline Dimensión & Variable & $\begin{array}{c}\text { Operacionaliza- } \\
\text { ción }\end{array}$ & $\begin{array}{c}\text { Primer } \\
\text { semestre }\end{array}$ & $\begin{array}{c}\text { Quinto } \\
\text { semestre }\end{array}$ & $\begin{array}{l}\text { Décimo } \\
\text { semestre }\end{array}$ \\
\hline \multirow{10}{*}{ Demográficas } & \multirow{2}{*}{ Edad } & \multirow{2}{*}{ En años } & $=21.20$ & $=21.97$ & $=25.22$ \\
\hline & & & $\delta=4.68$ & $\delta=4.99$ & $\delta=3.52$ \\
\hline & \multirow{2}{*}{ Género } & Masculino & $52,7 \%$ & $43,2 \%$ & $48,4 \%$ \\
\hline & & Femenino & $47,3 \%$ & $56,7 \%$ & $51,6 \%$ \\
\hline & \multirow{2}{*}{$\begin{array}{l}\text { Personas a } \\
\text { cargo }\end{array}$} & Número de & $=0.63$ & $=0.27$ & $=0.48$ \\
\hline & & personas a cargo & $\delta=0.32$ & $\delta=0.5$ & $\delta=0.75$ \\
\hline & \multirow{4}{*}{$\begin{array}{l}\text { Estado } \\
\text { civil }\end{array}$} & Soltero & $90,9 \%$ & $88,9 \%$ & $67,7 \%$ \\
\hline & & Casado & $1,8 \%$ & $0,0 \%$ & $6,5 \%$ \\
\hline & & Unión libre & $5,5 \%$ & $8,3 \%$ & $25,8 \%$ \\
\hline & & Divorciado & $1,8 \%$ & $2,8 \%$ & $0,0 \%$ \\
\hline \multirow{9}{*}{ Sociales } & \multirow{4}{*}{$\begin{array}{l}\text { Tipo de } \\
\text { vivienda }\end{array}$} & Propio & $45,5 \%$ & $32,4 \%$ & $32,3 \%$ \\
\hline & & En arriendo & $25,5 \%$ & $45,9 \%$ & $38,7 \%$ \\
\hline & & Familiar & $27,3 \%$ & $21,6 \%$ & $29,0 \%$ \\
\hline & & Otro & $1,8 \%$ & $0,0 \%$ & $0,0 \%$ \\
\hline & \multirow{2}{*}{$\begin{array}{l}\text { Años de } \\
\text { educación }\end{array}$} & \multirow{2}{*}{$\begin{array}{l}\text { Medida en años } \\
\text { de educación }\end{array}$} & $=12.83$ & $=15.16$ & $=16.77$ \\
\hline & & & $\delta=1.69$ & $\delta=1.7$ & $\delta=0.97$ \\
\hline & \multirow{3}{*}{ Ocupación } & ¿Cuál es su & & & \\
\hline & & ocupación? & $21,8 \%$ & $40,5 \%$ & $87 \%$ \\
\hline & & $\begin{array}{l}\text { Estudiante } \\
\text { Empleado }\end{array}$ & $78,2 \%$ & $59,5 \%$ & $13 \%$ \\
\hline \multirow{7}{*}{ Económicas } & & Medido & & & \\
\hline & mensual & $\begin{array}{l}\text { en pesos } \\
\text { colombianos }\end{array}$ & $\delta=\$ 1.272 .727$ & $\begin{array}{c}=864.865 \\
\delta=741.152\end{array}$ & $\begin{array}{l}=1.438 .8 / 0 \\
\delta=712.603\end{array}$ \\
\hline & \multirow{5}{*}{$\begin{array}{l}\text { Estrato } \\
\text { socioeco- } \\
\text { nómico }\end{array}$} & Estrato 1 & $1,9 \%$ & $2,7 \%$ & $3,2 \%$ \\
\hline & & Estrato 2 & $33,3 \%$ & $40,5 \%$ & $35,5 \%$ \\
\hline & & Estrato 3 & $48,1 \%$ & $51,4 \%$ & $58,1 \%$ \\
\hline & & Estrato 4 & $14,8 \%$ & $5,4 \%$ & $3,2 \%$ \\
\hline & & Estrato 5 & $1,9 \%$ & $0,0 \%$ & $0,0 \%$ \\
\hline
\end{tabular}

Elaboración propia. 
Tabla 2.

Descriptivos Variables Cualitativas

\begin{tabular}{|c|c|c|c|c|}
\hline Categoría & Operacionalización & $\begin{array}{c}\text { Primer } \\
\text { semestre }\end{array}$ & $\begin{array}{c}\text { Quinto } \\
\text { semestre }\end{array}$ & $\begin{array}{l}\text { Décimo } \\
\text { semestre }\end{array}$ \\
\hline \multirow{34}{*}{$\begin{array}{l}\text { Interacción } \\
\text { social }\end{array}$} & $\begin{array}{l}\text { Cuando usted conoce a una persona que tiene } \\
\text { más dinero que usted, usted piensa: }\end{array}$ & & & \\
\hline & & $5,8 \%$ & $2,8 \%$ & $11,5 \%$ \\
\hline & - Debe ser amigo mío & $5,8 \%$ & $2,8 \%$ & $3,8 \%$ \\
\hline & - Debe ser muy feliz & $0,0 \%$ & $2,8 \%$ & $0,0 \%$ \\
\hline & - Debe ser un déspota & $7,7 \%$ & $22,2 \%$ & $0,0 \%$ \\
\hline & - Debe tener muchas cosas que usted querria & $1,9 \%$ & $2,8 \%$ & $7,7 \%$ \\
\hline & - Es más inteligente que usted & $0,0 \%$ & $8,3 \%$ & $0,0 \%$ \\
\hline & - Es mejor que usted & $40,4 \%$ & $30,6 \%$ & $53,8 \%$ \\
\hline & - Ha tenido mas suerte que usted & $7,7 \%$ & $11,1 \%$ & $0,0 \%$ \\
\hline & $\begin{array}{l}\text { - Ha trabajado para conseguirio } \\
\text { - Nació con dinero }\end{array}$ & $11,5 \%$ & $5,6 \%$ & $3,8 \%$ \\
\hline & - Nacio con anero & $0,0 \%$ & $5,6 \%$ & $0,0 \%$ \\
\hline & $\begin{array}{l}\text { - Ha sabido invertir } \\
\text { - Otro }\end{array}$ & $19,2 \%$ & $5,6 \%$ & $19,2 \%$ \\
\hline & $\begin{array}{l}\text { Cuando usted conoce a una persona que tiene } \\
\text { menos dinero que usted, usted piensa: }\end{array}$ & & & \\
\hline & - Debe estar muy triste & $1,9 \%$ & $0,0 \%$ & $0,0 \%$ \\
\hline & - Debe pasar muchas necesidades & $13,5 \%$ & $27,8 \%$ & $29,0 \%$ \\
\hline & - Es peor que usted & $1,9 \%$ & $0,0 \%$ & $0,0 \%$ \\
\hline & - Es un fracasado & $\begin{array}{l}5,0 \% \\
21,2 \%\end{array}$ & $\begin{array}{c}0,0 \% \\
11.1 \%\end{array}$ & $\begin{array}{c}0,0 \% \\
12,9 \%\end{array}$ \\
\hline & - Es una persona con pocas ambiciones & $36,5 \%$ & $52,8 \%$ & $45,2 \%$ \\
\hline & $\begin{array}{l}\text { - No tuvo oportunidades } \\
\text { - Otro }\end{array}$ & $21,2 \%$ & $8,3 \%$ & $12,9 \%$ \\
\hline & Desea tener amigos con dinero: & & & \\
\hline & - Totalmente de acuerdo & $0,0 \%$ & $2,8 \%$ & $3,3 \%$ \\
\hline & - Parcialmente de acuerdo & $5,5 \%$ & $8,3 \%$ & $0,0 \%$ \\
\hline & - Parcialmente en desacuerdo & $\begin{array}{l}21,8 \% \\
72,7 \%\end{array}$ & $\begin{array}{l}22,2 \% \\
66,7 \%\end{array}$ & $\begin{array}{l}33,3 \% \\
63,3 \%\end{array}$ \\
\hline & - En desacuerdo & & & \\
\hline & $\begin{array}{l}\text { Se casaría (casó) con una persona con menos } \\
\text { dinero que usted: }\end{array}$ & & & \\
\hline & - Totalmente de acuerdo & $70,9 \%$ & $63,9 \%$ & $50,0 \%$ \\
\hline & - Parcialmente de acuerdo & $16,4 \%$ & $25,0 \%$ & $26,7 \%$ \\
\hline & - Parcialmente en desacuerdo & $10,9 \%$ & $11,1 \%$ & $16,7 \%$ \\
\hline & - En desacuerdo & $1,8 \%$ & $0,0 \%$ & $6,7 \%$ \\
\hline & $\begin{array}{l}\text { Formaría un negocio con alguien con menos } \\
\text { dinero que usted: }\end{array}$ & & & \\
\hline & & $47,3 \%$ & $41,7 \%$ & $33,3 \%$ \\
\hline & - Parcialmente de acuerdo & $34,5 \%$ & $38,9 \%$ & $43,3 \%$ \\
\hline & - Parcialmente en desacuerdo & $14,5 \%$ & $16,7 \%$ & $20,0 \%$ \\
\hline & - En desacuerdo & $3,6 \%$ & $2,8 \%$ & $3,3 \%$ \\
\hline
\end{tabular}




\begin{tabular}{|c|c|c|c|c|}
\hline Categoría & Operacionalización & $\begin{array}{c}\text { Primer } \\
\text { semestre }\end{array}$ & $\begin{array}{l}\text { Quinto } \\
\text { semestre }\end{array}$ & $\begin{array}{l}\text { Décimo } \\
\text { semestre }\end{array}$ \\
\hline \multirow{30}{*}{$\begin{array}{l}\text { Compor- } \\
\text { tamiento } \\
\text { ético }\end{array}$} & $\begin{array}{l}\text { La ley debe cumplirse así no se esté de } \\
\text { acuerdo con ella: }\end{array}$ & & & \\
\hline & - Totalmente de acuerdo & $41,8 \%$ & $64,9 \%$ & $50,0 \%$ \\
\hline & - Parcialmente de acuerdo & $25,5 \%$ & $16,2 \%$ & $33,3 \%$ \\
\hline & - Parcialmente en desacuerdo & $20,0 \%$ & $5,4 \%$ & $10,0 \%$ \\
\hline & - En desacuerdo & $12,7 \%$ & $13,5 \%$ & $6,7 \%$ \\
\hline & El fin justifica los medios: & & & \\
\hline & - Totalmente de acuerdo & $8,0 \%$ & $5,7 \%$ & $3,4 \%$ \\
\hline & - Parcialmente de acuerdo & $32,0 \%$ & $11,4 \%$ & $20,7 \%$ \\
\hline & - Parcialmente en desacuerdo & $24,0 \%$ & $34,3 \%$ & $24,1 \%$ \\
\hline & - En desacuerdo & $36,0 \%$ & $48,6 \%$ & $51,7 \%$ \\
\hline & Todo el mundo tiene precio: & & & \\
\hline & - Totalmente de acuerdo & $14,8 \%$ & $2,8 \%$ & $20,0 \%$ \\
\hline & - Parcialmente de acuerdo & $14,8 \%$ & $22,2 \%$ & $10,0 \%$ \\
\hline & - Parcialmente en desacuerdo & $11,1 \%$ & $27,8 \%$ & $16,7 \%$ \\
\hline & - En desacuerdo & $59,3 \%$ & $47,2 \%$ & $53,3 \%$ \\
\hline & $\begin{array}{l}\text { Si se da cuenta de que su jefe comete un acto } \\
\text { de corrupción usted lo denuncia: }\end{array}$ & & & \\
\hline & - Totalmente de acuerdo & $56,4 \%$ & $51,4 \%$ & $60,0 \%$ \\
\hline & - Parcialmente de acuerdo & $25,5 \%$ & $35,1 \%$ & $40,0 \%$ \\
\hline & - Parcialmente en desacuerdo & $14,5 \%$ & $8,1 \%$ & $0,0 \%$ \\
\hline & - En desacuerdo & $3,6 \%$ & $5,4 \%$ & $0,0 \%$ \\
\hline & $\begin{array}{l}\text { Si tuviera la oportunidad de robarse } 50.000 \\
\text { millones de pesos lo haría sin dudarlo: }\end{array}$ & & & \\
\hline & - Totalmente de acuerdo & $3,6 \%$ & $0,0 \%$ & $3,4 \%$ \\
\hline & - Parcialmente de acuerdo & $1,8 \%$ & $0,0 \%$ & $0,0 \%$ \\
\hline & - Parcialmente en desacuerdo & $12,7 \%$ & $2,7 \%$ & $17,2 \%$ \\
\hline & - En desacuerdo & $81,8 \%$ & $97,3 \%$ & $79,3 \%$ \\
\hline & Cuando usted se equivoca intenta ocultarlo: & & & \\
\hline & - Totalmente de acuerdo & $3,6 \%$ & $2,8 \%$ & $10,0 \%$ \\
\hline & - Parcialmente de acuerdo & $14,5 \%$ & $25,0 \%$ & $16,7 \%$ \\
\hline & - Parcialmente en desacuerdo & $30,9 \%$ & $27,8 \%$ & $30,0 \%$ \\
\hline & - En desacuerdo & $50,9 \%$ & $44,4 \%$ & $43,3 \%$ \\
\hline
\end{tabular}




\begin{tabular}{|c|c|c|c|c|}
\hline Categoría & Operacionalización & $\begin{array}{l}\text { Primer } \\
\text { semestre }\end{array}$ & $\begin{array}{l}\text { Quinto } \\
\text { semestre }\end{array}$ & $\begin{array}{l}\text { Décimo } \\
\text { semestre }\end{array}$ \\
\hline \multirow{8}{*}{$\begin{array}{l}\text { Obtención } \\
\text { y adminis- } \\
\text { tración de } \\
\text { rentas }\end{array}$} & $\begin{array}{l}\text { Usted ha aprendido habilidades importantes } \\
\text { para obtener dinero de: }\end{array}$ & & & \\
\hline & $\begin{array}{l}\text { - La escuela, el colegio, la universidad } \\
\text { - Otros familiares } \\
\text { - Su jefe } \\
\text { - Sus amigos } \\
\text { - Sus padres }\end{array}$ & $\begin{array}{c}13,5 \% \\
3,8 \% \\
11,5 \% \\
1,9 \% \\
69,2 \% \\
\end{array}$ & $\begin{array}{c}47,2 \% \\
0,0 \% \\
5,6 \% \\
2,8 \% \\
44,4 \% \\
\end{array}$ & $\begin{array}{c}23,3 \% \\
13,3 \% \\
16,7 \% \\
3,3 \% \\
43,3 \% \\
\end{array}$ \\
\hline & Usted considera que para obtener dinero debe: & & & \\
\hline & $\begin{array}{l}\text { - Asociarse } \\
\text { - Ayudar a los demás } \\
\text { - Estudiar } \\
\text { - Hacer negocios (comerciar) } \\
\text { - Otro } \\
\text { - Producir bienes o prestar servicios } \\
\text { - Tener propiedades que renten } \\
\text { - Trabajar }\end{array}$ & $\begin{array}{c}3,7 \% \\
1,9 \% \\
11,1 \% \\
16,7 \% \\
1,9 \% \\
1,9 \% \\
14,8 \% \\
48,1 \% \\
\end{array}$ & $\begin{array}{c}2,8 \% \\
0,0 \% \\
19,4 \% \\
22,2 \% \\
0,0 \% \\
8,3 \% \\
11,1 \% \\
36,1 \% \\
\end{array}$ & $\begin{array}{c}3,2 \% \\
0,0 \% \\
0,0 \% \\
22,6 \% \\
0,0 \% \\
12,9 \% \\
9,7 \% \\
51,6 \% \\
\end{array}$ \\
\hline & Usted desea tener mucho dinero & & & \\
\hline & $\begin{array}{l}\text { - Totalmente de acuerdo } \\
\text { - Parcialmente de acuerdo } \\
\text { - Parcialmente en desacuerdo } \\
\text { - En desacuerdo }\end{array}$ & $\begin{array}{c}40,0 \% \\
43,6 \% \\
9,1 \% \\
7,3 \% \\
\end{array}$ & $\begin{array}{c}43,2 \% \\
51,4 \% \\
2,7 \% \\
2,7 \% \\
\end{array}$ & $\begin{array}{c}43,3 \% \\
50,0 \% \\
6,7 \% \\
0,0 \% \\
\end{array}$ \\
\hline & ¿Si tuviera mucho dinero que haría con él? & & & \\
\hline & $\begin{array}{l}\text { - Lo invertiría } \\
\text { - Lo gastaría } \\
\text { - Otro } \\
\text { - Lo ahorraría }\end{array}$ & $\begin{array}{c}85,5 \% \\
9,1 \% \\
1,8 \% \\
3,6 \%\end{array}$ & $\begin{array}{l}89,2 \% \\
0,0 \% \\
8,1 \% \\
2,7 \%\end{array}$ & $\begin{array}{c}93,5 \% \\
6,5 \% \\
0,0 \% \\
0,0 \%\end{array}$ \\
\hline
\end{tabular}

Elaboración propia.

El resultado del análisis de correspondencia para toda la información recolectada mostró que las variables de edad, años de educación y estrato socioeconómico contribuían significativamente a explicar las categorías.

Por ejemplo, en la categoría "obtención y administración de rentas", tres preguntas (cómo aprendió a obtener rentas, qué habilidades considera importantes para este fin, y cómo administraría su dinero) se presentan como representativas a la hora de explicar las variables socioeconómicas. Al mismo tiempo, en la categoría "comportamiento ético", las respuestas sobre aceptación 
de equivocaciones, actuación ética y tolerancia a la corrupción se asocian de forma importante con esas variables.

En la categoría "interacción social" son importantes las preguntas asociadas a formar un negocio o casarse con alguien en un nivel social más bajo, y al deseo de tener amigos en posiciones sociales más elevadas.

Al analizar la variable edad con relación a las creencias exploradas, no se encontró asociación clara entre un rango de edad específico y alguna respuesta o categoría en particular. Mientras que al analizar la variable estrato socioeconómico, fue posible explicar los datos en cuatro dimensiones (Figura 1). La dimensión $1(40,01 \%)$ nos muestra una persona altamente tolerante a la corrupción y con habilidades para asociarse fácilmente. La dimensión 2 $(26,66 \%)$ describe a una persona que tiende a ahorrar. La dimensión $3(23,04 \%)$ tiene dos características relevantes: la persona no se casaría con otra que tenga menos recursos, y si comete una equivocación tiende a ocultarla. Por último, la dimensión $4(10,29 \%)$ tiene que ver con la obtención de dinero por medio de otras acciones, como invertir apropiadamente los ingresos.

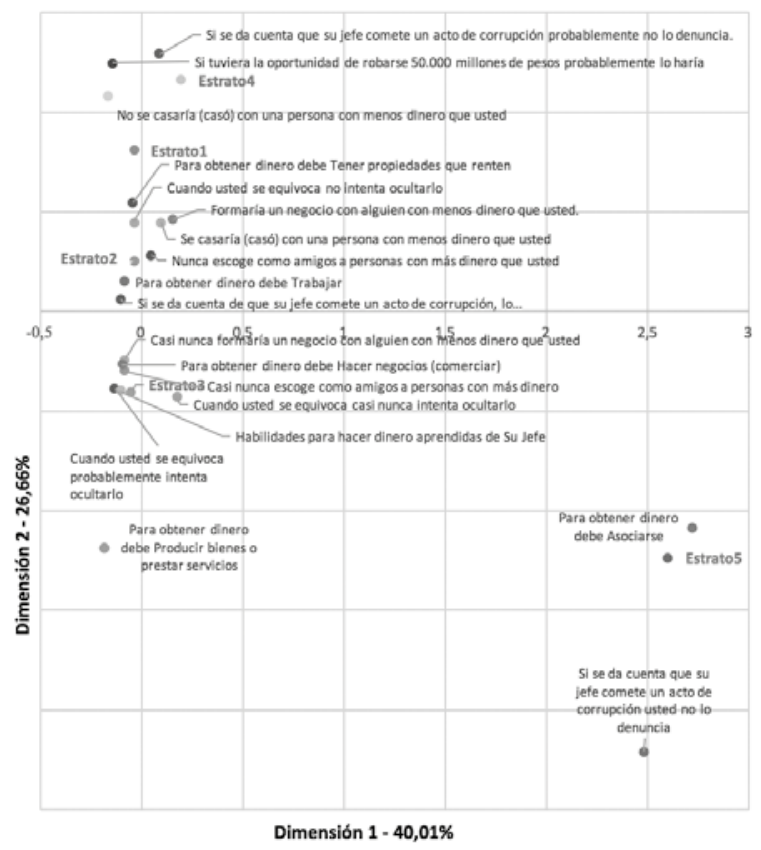

Figura 1. Asociaciones identificadas en el análisis de correspondencia por estrato. Elaboración propia. 


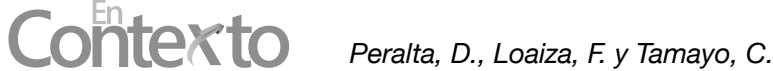

De esta forma se observa que los estudiantes de los estratos 4 y 5 presentan mayor nivel de tolerancia a la corrupción que los de los estratos 1,2 y 3 . Por otro lado, los estudiantes de los estratos 1 y 4 creen que ahorrar es importante mientras que aquellos de los estratos 3 y 5 no le dan mucha importancia.

Es evidente que hay claros niveles de asociación entre las categorías de creencias analizadas y los estratos socioeconómicos de los estudiantes, y esto permite establecer tendencias hacia actitudes de tipos específicos de individuos, por ejemplo, para los estudiantes de estratos 1 y 2 no es importante la interacción social, mientras que asociarse y producir bienes y servicios son habilidades relevantes para los estudiantes de estrato 5, como se observa en la Tabla 3.

Tabla 3.

Creencias asociadas con el estrato

\begin{tabular}{|c|c|c|c|c|c|c|}
\hline Categoría & Creencia & E 1 & E 2 & E 3 & E 4 & E 5 \\
\hline \multirow{5}{*}{$\begin{array}{l}\text { Interacción } \\
\text { Social }\end{array}$} & $\begin{array}{l}\text { Casi nunca escoge como amigos a } \\
\text { personas con más dinero que usted }\end{array}$ & & & $\mathrm{X}$ & & \\
\hline & $\begin{array}{l}\text { Nunca escoge como amigos a } \\
\text { personas con más dinero que usted }\end{array}$ & & $\mathrm{X}$ & & & \\
\hline & $\begin{array}{l}\text { Formaría un negocio con alguien } \\
\text { con menos dinero que usted }\end{array}$ & & & & $\mathrm{X}$ & \\
\hline & $\begin{array}{l}\text { Casi nunca formaría un negocio con } \\
\text { alguien con menos dinero que usted }\end{array}$ & & & $\mathrm{X}$ & & \\
\hline & $\begin{array}{l}\text { No formaría un negocio con alguien } \\
\text { con menos dinero que usted }\end{array}$ & & & & & $\mathrm{X}$ \\
\hline \multirow{6}{*}{$\begin{array}{l}\text { Obtención y ad- } \\
\text { ministración de } \\
\text { rentas }\end{array}$} & Para obtener dinero debe asociarse & & & & & $\mathrm{X}$ \\
\hline & $\begin{array}{l}\text { Para obtener dinero debe hacer } \\
\text { negocios (comerciar) }\end{array}$ & & & $\mathrm{X}$ & & \\
\hline & $\begin{array}{l}\text { Para obtener dinero debe producir } \\
\text { bienes o prestar servicios }\end{array}$ & & & & & $\mathrm{X}$ \\
\hline & $\begin{array}{l}\text { Para obtener dinero debe tener } \\
\text { propiedades que renten }\end{array}$ & $\mathrm{X}$ & $\mathrm{X}$ & & & \\
\hline & Para obtener dinero debe trabajar & & $\mathrm{X}$ & & & \\
\hline & $\begin{array}{l}\text { Habilidades para hacer dinero } \\
\text { aprendidas de su jefe }\end{array}$ & & & $\mathrm{X}$ & & \\
\hline
\end{tabular}




\begin{tabular}{|c|c|c|c|c|c|c|}
\hline Categoría & Creencia & E 1 & E 2 & E 3 & E 4 & E 5 \\
\hline \multirow{7}{*}{$\begin{array}{l}\text { Comportamiento } \\
\text { ético }\end{array}$} & $\begin{array}{l}\text { Si se da cuenta de que su jefe } \\
\text { comete un acto de corrupción lo } \\
\text { denuncia }\end{array}$ & & $X$ & & & \\
\hline & $\begin{array}{l}\text { Si se da cuenta que de su jefe } \\
\text { comete un acto de corrupción } \\
\text { probablemente no lo denuncia }\end{array}$ & & & & $\mathrm{X}$ & \\
\hline & $\begin{array}{l}\text { Si se da cuenta de que su jefe } \\
\text { comete un acto de corrupción usted } \\
\text { no lo denuncia }\end{array}$ & & & & & $\mathrm{X}$ \\
\hline & $\begin{array}{l}\text { Si tuviera la oportunidad de } \\
\text { robarse } 50.000 \text { millones de pesos } \\
\text { probablemente lo haría }\end{array}$ & $X$ & & & $X$ & \\
\hline & $\begin{array}{l}\text { Cuando usted se equivoca } \\
\text { probablemente intenta ocultarlo }\end{array}$ & & & $X$ & & \\
\hline & $\begin{array}{l}\text { Cuando usted se equivoca casi } \\
\text { nunca intenta ocultarlo }\end{array}$ & & & & & $X$ \\
\hline & $\begin{array}{l}\text { Cuando usted se equivoca no } \\
\text { intenta ocultarlo }\end{array}$ & $\mathrm{X}$ & $\mathrm{X}$ & & & \\
\hline
\end{tabular}

Elaboración propia.

Las creencias que tienen mayor presencia en los estratos 4 y 5 son de personas altamente tolerantes a la corrupción e incluso capaces de cometer actos corruptos, con habilidades para asociarse fácilmente, no se casarían con personas con menos recursos económicos, creen que para obtener rentas se deben producir bienes o prestar servicios y presentan tendencia a ahorrar en lugar de gastar o invertir.

El análisis de correspondencias de la variable años de educación con relación a las creencias de los estudiantes, identificó cuatro dimensiones que aplican los datos. En la primera $(36,14 \%)$ se observa una persona que cree que debe asociarse, probablemente tener amigos con más dinero y gastar el dinero que gana en lugar de ahorrarlo o invertirlo. En la segunda dimensión $(27,42 \%)$, son personas que aprendieron a obtener rentas del colegio o la universidad y consideran importante prestar bienes o servicios. La tercera dimensión $(22,89 \%)$, si tuvieran mucho dinero tendrían comportamientos altruistas, como ayudar a sus familiares o dar regalos, aprendieron a obtener rentas del colegio o la universidad y cuando se equivocan probablemente intenten ocultarlo. Por 


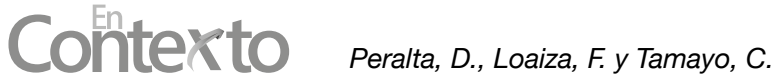

último, la cuarta dimensión (13,55\%), se enfoca en los individuos que creen que es poco probable casarse con una persona con menos recursos económicos $\mathrm{y}$ aprendieron de sus amigos a hacer dinero.

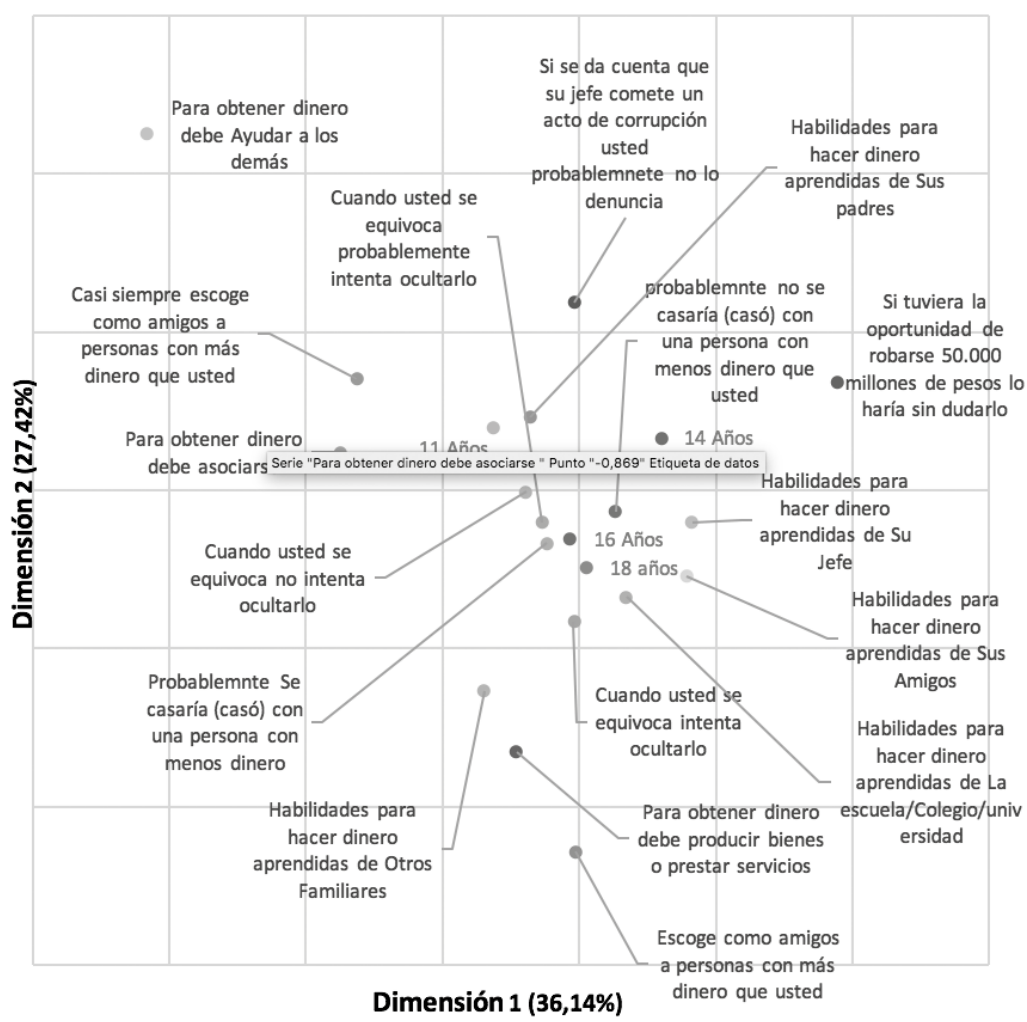

Figura 2. Correspondencias entre años de educación y creencias.

Elaboración propia.

Los individuos con 11 años de educación son estudiantes de primer semestre con educación secundaria finalizada, los estudiantes con 13 años de educación son aquellos que han recibido ciclos de educación técnica o tecnológica e ingresan a primer semestre. Los estudiantes con 14 años de educación son los que se encuentran cursando quinto semestre y no tienen formación técnica. El grupo de estudiantes con 16 años de educación incluye estudiantes de último semestre o aquellos que en quinto recibieron formación técnica. 
Al evaluar las distancias absolutas (ver Anexo 1) entre las coordenadas de los años de educación y las respuestas a las preguntas realizadas, encontramos que al comparar los estudiantes de menor educación con los de mayor nivel educativo, estos últimos escogían como amigos personas que tuvieran más recursos económicos que ellos, presentaban una leve mejoría en cuanto a: 1) la conducta ética, 2.) la formación de negocios con personas de otros niveles económicos, y 3) la capacidad de asociación.

Adicionalmente, en los estudiantes con mayor nivel educativo se observa una mayor cercanía con el aprendizaje de habilidades de otras personas para hacer dinero, cercanas a su núcleo social y diferentes de sus padres. De la misma forma, se identificaron consideraciones de ayuda a los demás, y la producción de bienes y servicios como camino para obtener rentas. No obstante lo anterior, los estudiantes más educados creen que es mejor ocultar sus equivocaciones y gastar en lugar de invertir o ahorrar. Desde el punto de vista de la tolerancia a la corrupción y la selección de pareja, la educación de la universidad parece no influir en sus creencias.

Por otro lado, al comparar los datos de los estudiantes con 16 y 18 años de educación, no se encontraron diferencias importantes, es decir, para los estudiantes que finalizan sus estudios de pregrado con o sin formación técnica no hay diferencias en sus creencias sobre las categorías evaluadas.

Sin embargo, para los estudiantes de primer semestre, se observa que aquellos que han adquirido formación técnica tienden a escoger amigos con más dinero, son levemente más tolerantes a la corrupción, pero con menor disposición a cometer estas conductas, intentan ocultar menos sus errores, tienden a ahorrar, privilegian el estudio y el comercio como formas para obtener ingresos y han aprendido habilidades de sus padres, además de su jefe y amigos, para la obtención de rentas.

\section{Discusión}

Como se observó en los resultados, las variables estrato y años de educación influyen en las creencias de los estudiantes, lo que es perfectamente lógico desde el punto de vista teórico, ya que en su gran mayoría los estudiantes continúan en el estrato de sus padres, donde recibieron la socialización primaria que configuró su realidad social y económica. En relación con los años de educación, ya se había anotado que estudios sobre movilidad social han identificado como factor importante la educación y/o superar el nivel 


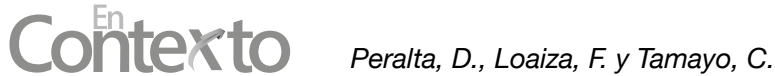

educativo de los padres. Adicionalmente, la universidad como institución brinda la socialización secundaria requerida para la interacción en lo que podría denominarse el submundo profesional de la formación contable.

En la categoría "interacción social" los elementos de mayor relevancia se referían a la escogencia de la pareja, los amigos y los posibles socios en un emprendimiento. A este respecto, se identificó que para los estudiantes de estratos socioeconómicos altos hay asociación entre su condición socioeconómica y los mencionados elementos, mientras que para los estudiantes de los estratos 1 y 2 se observaron asociaciones poco relevantes. Esta condición evidencia la necesidad de trabajar desde la Universidad en habilidades blandas que posibiliten un mejor desenvolvimiento de los estudiantes al relacionarse con personas de otras condiciones socioeconómicas.

En la categoría obtención y administración de rentas, se identificó que la creencia de los estudiantes con relación a cómo se debe obtener el dinero se asocia estrechamente con su estrato. De esta forma, los estratos bajos consideran que tener propiedades que renten y trabajar son las mejores formas para obtener rentas, mientras que para los estratos altos es fundamental la capacidad de asociación y la producción de bienes y servicios. El estrato 3 considera que dedicarse al comercio le permitirá obtener rentas.

En estos resultados se observa claramente la división del trabajo como una condición para explicar la posición socioeconómica, matizando en las creencias de los estudiantes las preferencias ordinales de la sociedad, en relación con premiar o desincentivar unas $\mathrm{u}$ otras actividades.

Para la categoría comportamiento ético se encontró que los estratos altos muestran tolerancia a la corrupción al creer que no deben denunciar a su jefe si saben que ha realizado un acto de corrupción, mientras que los estratos bajos consideran todo lo contrario. No obstante, los estratos 1 y 4 presentan tendencia a cometer actos corruptos, pues no dudan en apropiarse de rentas si se llegase el caso. El estrato 3, por su parte, muestra tendencia a ocultar sus equivocaciones, no obstante, la tolerancia a la corrupción o la tendencia a cometer un acto de corrupción no se muestran como elementos significativos.

Como complemento de lo anterior, se identificó que la educación superior de la Universidad de La Salle no está contribuyendo a reducir la tolerancia a la corrupción, aunque muestra un leve efecto hacia la disminución de las conductas corruptas. Se considera prioritario identificar herramientas para 
intervenir sobre este aspecto, teniendo en cuenta los altos niveles de percepción de corrupción, la función que la ley otorga a los contadores públicos como garantes de la confianza en los mercados y las desastrosas consecuencias que los actos corruptos tienen sobre la sociedad en su conjunto.

Sin embargo, la formación de la Universidad de La Salle sí contribuyó a mejorías en otros aspectos, cabe resaltar la incorporación de nociones altruistas de ayuda al prójimo a partir de la formación lasallista, la cual propende por el humanismo y la reflexión crítica, donde prevalecen experiencias formativas fundamentadas en valores para que las producciones de saber se conviertan en manifestaciones de sentido (Universidad de La Salle, 2008).

Este también es uno de los objetivos a los que la Universidad le debe apuntar, a la formación de jóvenes con criterio, capaces de pensar el mundo y actuar en él, tal como lo plantea Nussbaum, al referirse a las escuelas, pero que bien puede trasladarse a la universidad: la escuela puede desarrollar la capacidad de sentir interés genuino por los demás, promover activamente el pensamiento crítico, así como la habilidad y el coraje de expresarlo, aunque disienta de los demás. (Nussbaum, 2010).

\section{Conclusiones}

A mayores niveles de educación, la renta es más alta, el aumento es particularmente importante entre los estudiantes de $5 .^{\circ}$ y $10 .^{\circ}$ semestre, entre los que se identifica una variación positiva del $66 \%$ en el nivel de ingreso, y del $64,5 \%$ entre los estudiantes con 16 y 18 años de educación. Lo anterior confirma la relevancia de la función redistributiva de la educación.

Las variables de estrato socioeconómico y años de educación influyen en las creencias de los estudiantes con relación a la administración y obtención de rentas, esto indica que pueden construirse tendencias con relación a creencias que podrían influir en la movilidad social para la construcción de una estructura social igualitaria.

La identificación de las creencias relacionadas específicamente con cada estrato ofrece resultados preocupantes, pues se identifican la tolerancia a la corrupción y conductas éticas inapropiadas como características de los estudiantes de estratos altos. Estas características no las puede fortalecer la Universidad, en este sentido el proceso educativo no puede éticamente generar procesos de movilidad social desde las mencionadas creencias. 


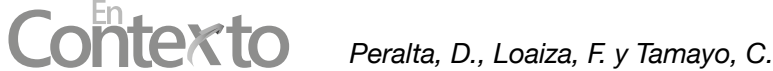

No obstante, se identificó que la educación superior puede potenciar actitudes positivas: la capacidad de asociación, el desarrollo de habilidades sociales para la interacción con individuos de otros niveles sociales, el emprendimiento para la producción de bienes o prestación de servicios, y la comprensión del ahorro como vía para acumular el capital que permita la inversión productiva.

Por último, en las creencias de los estudiantes se observa una expresión de la división del trabajo y las preferencias sociales, es así como la actividad rentística y el trabajo se asocian a los estratos 1 y 2 , el comercio al estrato $3, \mathrm{y}$ el ahorro, la capacidad de asociación y la producción de bienes y servicios, a los estratos 4 y 5 .

\section{Referencias}

Araya, S. (2002). Las representaciones sociales: ejes teóricos para su discusión. Costa Rica: Facultad Latinoamericana de Ciencias Sociales.

Arnau, M. (2010). Aportaciones sobre la relación conceptual. Electronic Journal of Research in Educational Psychology.

Bazdresch, M. (2001). Educación y pobreza: una relación conflictiva. Pobreza, desigualdad social y ciudadanía, 65-81. Recuperado de http://feae.eu/wp-content/uploads/2017/09/CLACSO-POBREZA-YEDUCACI\%C3\%93N.pdf

Berger, P. y Luckmann, T. (2003). La Construcción Social de la Realidad (Decimoctava impresion ed.). Buenos Aires, Argentina: Amorrortu editores.

Esteban, N. y Grillo, S. (2000). Educación, Movilidad Social y "Trampa de la Pobreza". Coyuntura Social, 101-119. Recuperado de http://hdl.handle. net/11445/1770

Lewin, K. (1978). La teoria del campo en la ciencia social. Argentina: Paidós.

Morales, F. (1999). Actitudes. En F. Morales, Psicologia social (pp. 194-205). España: MacGrawHill.

Musgrave, R. (1983). Public Finance, now and then*. Public Finance Analysis, $1-13$.

Nussbaum, M. (2010). Sin fines de lucro. Buenos Aires: Katz Editores.

Ostry, J., Berg, A. \& Tsangarides, C. (2014). Redistribution, Inequality and Growth. INTERNATIONAL MONETARY FUND. 
Ostry, J., Berg, A. y Tsangarides, C. (2014). Redistribución, Desigualdad y Crecimiento. Revista de Economía Institucional, 16(30), 53-81.

R Core Team. (2017). R: A language and environment for statistical computing. Recuperado de https://www.R-project.org/

Rios, J. (2002). Desigualdad ¿Qué Sabemos? Investigaciones Económicas, $X X V I(2), 221-254$.

Suarez, H. (2015). Actitud frente a la mercadotecnia en salud en los paises latinoamericanos. Horizonte Sanitario, 15(1), 23-28.

Universidad de La Salle. (2008). Enfoque Formativo Lasallista. Bogotá D.C.

\section{Anexo 1}

Distancias absolutas entre coordenadas (años de educación)

\begin{tabular}{|c|c|c|c|c|c|}
\hline Creencia & $\begin{array}{c}11 \\
\text { años }\end{array}$ & $\begin{array}{c}13 \\
\text { años }\end{array}$ & $\begin{array}{c}14 \\
\text { años }\end{array}$ & $\begin{array}{c}16 \\
\text { años }\end{array}$ & $\begin{array}{l}18 \\
\text { años }\end{array}$ \\
\hline $\begin{array}{l}\text { Usted escoge como amigos a personas con más dinero } \\
\text { que usted: Totalmente de acuerdo }\end{array}$ & 1,0 & 1,3 & 1,6 & 1,0 & 0,9 \\
\hline $\begin{array}{l}\text { Usted escoge como amigos a personas con más dinero } \\
\text { que usted: Parcialmente de acuerdo }\end{array}$ & 0,3 & 0,6 & 0,9 & 0,3 & 0,2 \\
\hline $\begin{array}{l}\text { Usted escoge como amigos a personas con más dinero } \\
\text { que usted: Parcialmente en desacuerdo }\end{array}$ & 0,1 & 0,3 & 0,7 & 0,0 & 0,0 \\
\hline $\begin{array}{l}\text { Usted escoge como amigos a personas con más dinero } \\
\text { que usted: En desacuerdo }\end{array}$ & 0,3 & 0,0 & 0,3 & 0,3 & 0,4 \\
\hline $\begin{array}{l}\text { Si se da cuenta que su jefe comete un acto de } \\
\text { corrupción usted lo denuncia: Totalmente de acuerdo }\end{array}$ & 0,1 & 0,2 & 0,5 & 0,1 & 0,2 \\
\hline $\begin{array}{l}\text { Si se da cuenta que su jefe comete un acto de } \\
\text { corrupción usted lo denuncia: Parcialmente de } \\
\text { acuerdo }\end{array}$ & 0,1 & 0,2 & 0,5 & 0,1 & 0,2 \\
\hline $\begin{array}{l}\text { Si se da cuenta que su jefe comete un acto de } \\
\text { corrupción usted lo denuncia: Parcialmente en } \\
\text { desacuerdo }\end{array}$ & 0,7 & 0,5 & 0,1 & 0,8 & 0,8 \\
\hline $\begin{array}{l}\text { Si se da cuenta que su jefe comete un acto de } \\
\text { corrupción usted lo denuncia: En desacuerdo }\end{array}$ & 0,0 & 0,2 & 0,5 & 0,1 & 0,1 \\
\hline $\begin{array}{l}\text { Si tuviera la oportunidad de robarse } 50.000 \text { millones } \\
\text { de pesos lo haría sin dudarlo: Totalmente de acuerdo }\end{array}$ & 1,4 & 1,2 & 0,8 & 1,5 & 1,5 \\
\hline $\begin{array}{l}\text { Si tuviera la oportunidad de robarse } 50.000 \text { millones } \\
\text { de pesos lo haría sin dudarlo: Parcialmente de acuerdo }\end{array}$ & 2,6 & 2,3 & 2,0 & 2,6 & 2,7 \\
\hline
\end{tabular}




\begin{tabular}{|c|c|c|c|c|c|}
\hline Creencia & $\begin{array}{c}11 \\
\text { años }\end{array}$ & $\begin{array}{c}13 \\
\text { años }\end{array}$ & $\begin{array}{c}14 \\
\text { años }\end{array}$ & $\begin{array}{c}16 \\
\text { años }\end{array}$ & $\begin{array}{c}18 \\
\text { años }\end{array}$ \\
\hline $\begin{array}{l}\text { Si tuviera la oportunidad de robarse } 50.000 \text { millones } \\
\text { de pesos lo haría sin dudarlo: Parcialmente } \\
\text { en desacuerdo }\end{array}$ & 0,2 & 0,5 & 0,8 & 0,2 & 0,1 \\
\hline $\begin{array}{l}\text { Si tuviera la oportunidad de robarse } 50.000 \text { millones } \\
\text { de pesos lo haría sin dudarlo: En desacuerdo }\end{array}$ & 0,1 & 0,1 & 0,5 & 0,2 & 0,2 \\
\hline $\begin{array}{l}\text { Cuando usted se equivoca intenta ocultarlo: } \\
\text { Totalmente de acuerdo }\end{array}$ & 0,3 & 0,6 & 0,9 & 0,3 & 0,2 \\
\hline $\begin{array}{l}\text { Cuando usted se equivoca intenta ocultarlo- } \\
\text { Parcialmente de acuerdo }\end{array}$ & 0,1 & 0,4 & 0,7 & 0,0 & 0,0 \\
\hline $\begin{array}{l}\text { Cuando usted se equivoca intenta ocultarlo: } \\
\text { Parcialmente en desacuerdo }\end{array}$ & 0,1 & 0,3 & 0,7 & 0,0 & 0,0 \\
\hline $\begin{array}{l}\text { Cuando usted se equivoca intenta ocultarlo: } \\
\text { En desacuerdo }\end{array}$ & 0,4 & 0,2 & 0,2 & 0,5 & 0,5 \\
\hline $\begin{array}{l}\text { Se casaría (casó) con una persona con menos dinero } \\
\text { que usted: Totalmente de acuerdo }\end{array}$ & 0,2 & 0,0 & 0,4 & 0,3 & 0,3 \\
\hline $\begin{array}{l}\text { Se casaría (casó) con una persona con menos dinero } \\
\text { que usted: Parcialmente de acuerdo }\end{array}$ & 0,2 & 0,4 & 0,8 & 0,1 & 0,1 \\
\hline $\begin{array}{l}\text { Se casaría (casó) con una persona con menos dinero } \\
\text { que usted: Parcialmente en desacuerdo }\end{array}$ & 0,2 & 0,1 & 0,4 & 0,3 & 0,3 \\
\hline $\begin{array}{l}\text { Se casaría (casó) con una persona con menos dinero } \\
\text { que usted: En desacuerdo }\end{array}$ & 0,2 & 0,1 & 0,4 & 0,2 & 0,3 \\
\hline $\begin{array}{l}\text { Formaría un negocio con alguien con menos dinero } \\
\text { que usted: Totalmente de acuerdo }\end{array}$ & 0,1 & 0,1 & 0,5 & 0,2 & 0,2 \\
\hline $\begin{array}{l}\text { Formaría un negocio con alguien con menos dinero } \\
\text { que usted: Parcialmente de acuerdo }\end{array}$ & 0,2 & 0,1 & 0,4 & 0,3 & 0,3 \\
\hline $\begin{array}{l}\text { Formaría un negocio con alguien con menos dinero } \\
\text { que usted: Parcialmente en desacuerdo }\end{array}$ & 0,1 & 0,3 & 0,7 & 0,0 & 0,0 \\
\hline $\begin{array}{l}\text { Formaría un negocio con alguien con menos dinero } \\
\text { que usted: En desacuerdo }\end{array}$ & 0,8 & 0,5 & 0,2 & 0,8 & 0,9 \\
\hline Si tuviera usted mucho dinero: Lo invertiría & 0,1 & 0,1 & 0,4 & 0,2 & 0,2 \\
\hline Si tuviera usted mucho dinero: Lo gastaría & 0,7 & 0,9 & 1,3 & 0,6 & 0,6 \\
\hline Si tuviera usted mucho dinero: Otro & 0,1 & 0,2 & 0,5 & 0,1 & 0,2 \\
\hline Si tuviera usted mucho dinero: Lo ahorraría & 0,8 & 0,6 & 0,2 & 0,9 & 0,9 \\
\hline Para obtener dinero debe: Asociarse & 0,6 & 0,9 & 1,2 & 0,6 & 0,5 \\
\hline Para obtener dinero debe: Ayudar a los demás & 0,3 & 0,6 & 0,9 & 0,3 & 0,2 \\
\hline Para obtener dinero debe: Estudiar & 0,5 & 0,2 & 0,1 & 0,6 & 0,6 \\
\hline Para obtener dinero debe: Hacer negocios (comerciar) & 0,3 & 0,0 & 0,3 & 0,3 & 0,4 \\
\hline Para obtener dinero debe: Otro & 2,6 & 2,3 & 2,0 & 2,6 & 2,7 \\
\hline
\end{tabular}




\begin{tabular}{lccccc}
\hline \multicolumn{1}{c}{ Creencia } & $\begin{array}{c}11 \\
\text { años }\end{array}$ & $\begin{array}{c}13 \\
\text { años }\end{array}$ & $\begin{array}{c}14 \\
\text { años }\end{array}$ & $\begin{array}{c}16 \\
\text { años }\end{array}$ & $\begin{array}{c}18 \\
\text { años }\end{array}$ \\
\hline $\begin{array}{l}\text { Para obtener dinero debe: Producir bienes o prestar } \\
\text { servicios }\end{array}$ & 0,9 & 1,2 & 1,5 & 0,9 & 0,8 \\
\hline Para obtener dinero debe: Tener propiedades que renten & 0,6 & 0,4 & 0,1 & 0,7 & 0,7 \\
\hline Para obtener dinero debe: Trabajar & 0,0 & 0,3 & 0,6 & 0,0 & 0,1 \\
\hline $\begin{array}{l}\text { Habilidades para hacer dinero aprendidas de: } \\
\text { La escuela, el colegio, la universidad }\end{array}$ & 0,0 & 0,3 & 0,6 & 0,0 & 0,1 \\
\hline $\begin{array}{l}\text { Habilidades para hacer dinero aprendidas de: } \\
\text { Otros familiares }\end{array}$ & 0,9 & 1,1 & 1,4 & 0,8 & 0,8 \\
\hline Habilidades para hacer dinero aprendidas de: Su jefe & 0,4 & 0,2 & 0,2 & 0,5 & 0,5 \\
\hline $\begin{array}{l}\text { Habilidades para hacer dinero aprendidas de: } \\
\text { Sus amigos }\end{array}$ & 0,2 & 0,0 & 0,3 & 0,3 & 0,3 \\
\hline $\begin{array}{l}\text { Habilidades para hacer dinero aprendidas de: } \\
\text { Sus padres }\end{array}$ & 0,2 & 0,1 & 0,4 & 0,2 & 0,3 \\
\hline
\end{tabular}

Elaboración propia.

\section{Para citar este artículo:}

Peralta, D., Loaiza, F. y Tamayo, C. (2019). Movilidad Social y Educación. Creencias sobre obtención y administración de rentas asociadas a las variables socioeconómicas de los estudiantes de Contaduría Pública de la Universidad de La Salle. En-Contexto, 7(10), 89-111. 